\title{
Identification of Indica-Japonica Subspecies Rice by Duplex Fluorescence PCR Detection for Chloroplast DNA and Endogenous gos Gene
}

\author{
Biying Shao, Tingyu Miao, Juan Peng, Bin Chen, Wenbing Chen* \\ Technology Center of Fuzhou Customs District, Fuzhou, China \\ Email address: \\ byshao@souho.com (Biying Shao), miaoty419@163.com (Tingyu Miao),617572834@qq.com (Juan Peng), \\ 621213wbc@163.com (Wenbing Chen) \\ ${ }^{*}$ Corresponding author
}

\section{To cite this article:}

Biying Shao, Tingyu Miao, Juan Peng, Bin Chen, Wenbing Chen. Identification of Indica-Japonica Subspecies Rice by Duplex Fluorescence PCR Detection for Chloroplast DNA and Endogenous gos Gene. International Journal of Nutrition and Food Sciences.

Vol. 10, No. 5, 2021, pp. 101-107. doi: 10.11648/j.ijnfs.20211005.12

Received: August 17, 2021; Accepted: September 6, 2021; Published: September 23, 2021

\begin{abstract}
Asia cultivated rice are classified into two subspecies, indica and japonica rice (hsien and keng rice called in China, separately). It is necessary to establish a convenient and effective method to identify two subspecies because of the different quality characteristics between the rice products of them, which lead to different processing uses and commodity values. The identification method of PCR based on the difference of chloroplast DNA is convenient and effective, to be attempted to establish. Based on 69 bp fragment deletion of chloroplast DNA (cpDNA) found in ORF100 region nucleotides within the cpDNA Pst I-12 fragment in indica rice but not in japonica, the primer pair and a probe located on the specific cpDNA fragment in japonica rice were designed to identify indica and japonica rice. Another primer pair and probe used to detect endogenous gene gos in rice were combined with above primer pair and probe for detecting cpDNA to establish duplex fluorescence PCR to amplify cpDNA and gos gene for improving detection accuracy, to avoid the false negative results caused by DNA extraction error. The duplex fluorescence PCR detection method was established using typical japonica rice (pearl rice) and typical indica rice (Taixian 11). The accuracy of the method was validated with 547 samples including 177 samples of rice seed and eaten rice known as conventional japonica varieties and japonica type hybrid combinations and 370 samples of rice seed and eaten rice known as conventional indica rice varieties and indica type hybrid combinations. In 177 japonica samples, 170 samples with both positive results of cpDNA marker and gos gene, a coincidence rate of $96.05 \%$ agreement japonica rice, and 7 samples with positive results of gos gene and negative of cpDNA marker, not agreement japonica rice with non-coincidence rate of 3.95\%, were detected. Of the 370 samples of indica rice seed and eaten rice, with positive results of gos gene and negative of cpDNA marker were detected in 340 samples, the rate of coincidence to indica rice was $91.89 \%$. Other 30 samples with both positive results of cpDNA marker and gos gene, were detected, and the non-coincidence rate with indica rice was $8.11 \%$. These identification results were in good agreement with known indica and japonica varieties, and the coincidence rate of japonica varieties was higher than indica. This method can be used to identify indica and japonica subspecies rice, especially suitable to identify conventional rice varieties.
\end{abstract}

Keywords: Indica (hsien) Rice Seed/Eaten Rice, japonica (keng) Rice/Eaten Rice, Chloroplast DNA (cpDNA), Endogenous gos Gene, Duplex Fluorescence PCR, Identification

\section{Introduction}

Cultivated rices in Asia were divided into Oryza sativa L. subspecies indica and Oryza sativa L. subspecies japonica [1], hsien and keng subspecies also called in China for thousands of years [2,3]. The traditional identification methods of them are mainly morphological identification and biochemical index classification methods [1-7]. These methods have the defects of intermediate transition type and can not be clearly divided into two categories. With the development of molecular biology technology, more and more molecular markers, such as isozyme markers [7-8] and 
DNA markers including RFLP [9-11], RAPD [12, 13], InDel (insertion/deletion, japonica specific insertion deletion) [1418], cytoplasmic molecular marker [7, 19-23], were used to study the origin and the differentiation of indica-japonica subspecies in Asian cultivated rice (Oryza sativa L.).

It is necessary to establish a DNA identification method to distinguish indica rice and japonica rice because of their different commercial value. Kanno et al. [23] found that indica rice had a 69 bp deletion in the ORF100 region of Pst I-12 fragment of chloroplast DNA (cpDNA, same below) compared with japonica rice. Because of long-term natural or artificial hybridization, frequent communication of genetic information and complex DNA components in nucleus, it is difficult to identify indica-japonica subspecies based on differential markers of nuclear DNA. As a complicated method for the identification of indica-japonica subspecies, the previously published methods for the differentiation and identification of indica-japonica rice based on the PCR amplification polymorphism of nuclear DNA and cpDNA were presented, there are some problems such as expensive cost and low accuracy. This technique only detects one stable cpDNA site, which is accurate, efficient, economical and simple. Duplex fluorescence real-time PCR were widely used to detect the pathogenic bacteria and viruses in food [24-26] and plants [27], also the animal-derived components and genetically modified components in food [28-30]. There was no report on the identification of indica-japonica subspecies by duplex fluorescence real-time PCR. The primer pair selected (Chen. et al) and a probe located on the $69 \mathrm{bp}$ fragment in cpDNA of japonica rice were designed to identify indica and japonica rice [31]. Another primer pair and probe used to detect endogenous gene gos [32] of rice were combined with above primer pair and probe detecting cpDNA to establish duplex fluorescence PCR for cpDNA and gos gene for improving detection accuracy.

The accuracy of the method was validated with 177 samples of rice seed and eaten rice of japonica varieties and japonica hybrid combinations and 370 samples of rice seed and eaten rice of conventional indica rice varieties and indica hybrid combinations, and the reasons for non-coincidence were analyzed.

\section{Materials and Methods}

\subsection{Materials}

Rice seed and eaten rice were mainly collected from rice seeds consigned for inspection, eaten rice from local supermarkets in Fuzhou city, and local rice varieties and hybrid rice combinations from institutions such as Fujian Academy of Agricultural Sciences, Guangxi Academy of Agricultural Sciences, Yunnan Agricultural University and Kaili College in Guizhou Province, a total of 547 samples. The typical japonica pearl rice from Northeast China and typical indica rice Taixian 11 from Taiwan Province of China were used to sequence the specific cpDNA fragments of indica-japonica subspecies and to establish the duplex fluorescence PCR method.

\subsection{DNA Extraction and Duplex Fluorescence PCR}

\subsubsection{DNA Extraction}

$100 \mathrm{~g}$ rice seeds or eaten rice were ground into powder, 1.0 $\mathrm{g}$ of powder was added into $50 \mathrm{~mL}$ centrifuge tube, then CTAB method [31] was used to extract DNA. $2 \mu \mathrm{L}$ DNA solution were taken in the ultra-micro nucleotides determinator for examining, dilute to $100 \mathrm{ng} / \mu \mathrm{L}$ with $\mathrm{TE}$ solution ( $\mathrm{pH} 8.0$ ), keep at $-20^{\circ} \mathrm{C}$.

\subsubsection{Primer and Probe}

The sequence of primers and probes are shown in Table 1, the probe to be designed basing on the sequences on indicajaponica specific cpDNA fragment of rice in Figure 1. The PCR amplification system with 2 short target fragments and 5 ratios of primer dosage are shown in Table 2 .

Table 1. The sequences of primers and probes in duplex fluorescence PCR for cpDNA marker and endogenous gos gene to identify indica-japonica rice.

\begin{tabular}{lll}
\hline Target gene fragment & Primers & Primer sequences (5'- -3') \\
\hline \multirow{2}{*}{$\begin{array}{l}\text { indica-japonica } \text { specific } \\
\text { cpDNA segment of rice }\end{array}$} & Rice-F4 forward primer ${ }^{[31]}$ & AATCGCAACCCCTTTCCGC \\
& Rice-R1 reverse primer ${ }^{[31]}$ & TTGAGGATTATTCCATGATTCC \\
& gobe & HEX-ATGCAATAGAGAGCGAGTGG-BHQ1 \\
Endogenous gos gene & gos9-R1 reverse primer & TTAGCCTCCCGCTGCAGA \\
fragment in rice [32] & AGAGTCCACAAGTGCTCCCG \\
\hline
\end{tabular}

Table 2. The different proportion of two set primers and probes in duplex fluorescence PCR system for cpDNA marker and endogenous gos gene to identify indica-japonica rice.

\begin{tabular}{lllll}
\hline \multirow{2}{*}{ Composition } & \multicolumn{1}{l}{ Amount of addition $(\boldsymbol{\mu L})$} & & \\
\cline { 2 - 5 } & $\mathbf{1 : 1}$ & $\mathbf{1 : 2}$ & $\mathbf{1 : 3}$ & $\mathbf{1 : 4}$ \\
\hline $\mathrm{ddH}{ }_{2} \mathrm{O}$ & 8 & 7.5 & 7 & 6.5 \\
$2 \times$ Mix & 10 & 10 & 10 & 10 \\
gos-F & 0.2 & 0.2 & 0.2 & 0.2 \\
gos-R & 0.2 & 0.2 & 0.2 & 0.2 \\
gos-P & 0.1 & 0.1 & 0.1 & 0.1 \\
Rice-F4 & 0.2 & 0.4 & 0.6 & 0.8 \\
Rice-R1 & 0.2 & 0.4 & 0.6 & 0.1 \\
Rice-P & 0.1 & 0.2 & 0.3 & 1.0 \\
DNA $(200 \mathrm{ng} / \mu \mathrm{L})$ & 1 & 1 & 1 & 0.4 \\
\hline
\end{tabular}


F4

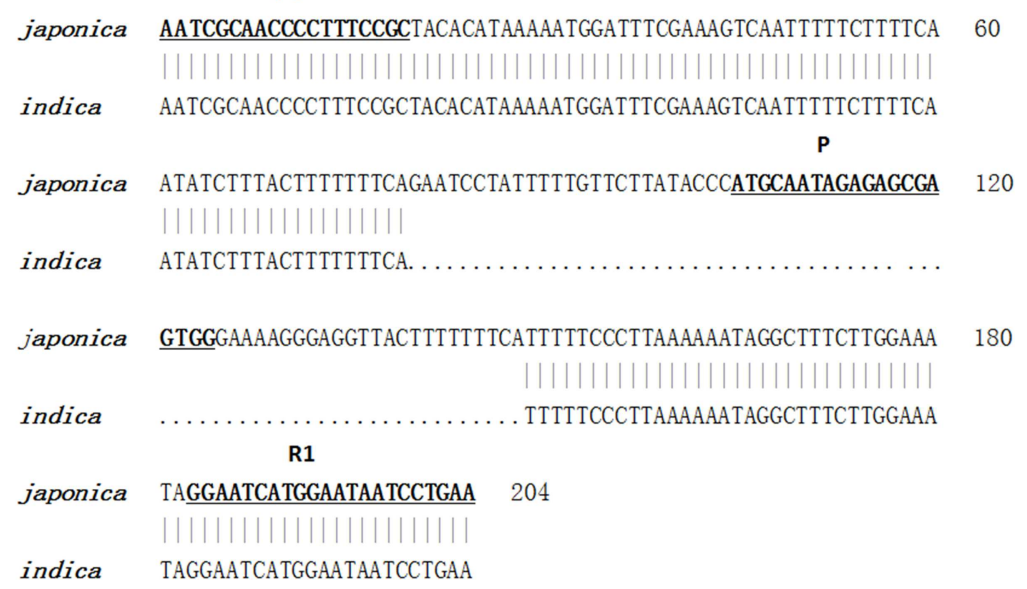

Figure 1. The position of Taqman fluorescence PCR probe in specific cpDNA fragment of japonica rice.

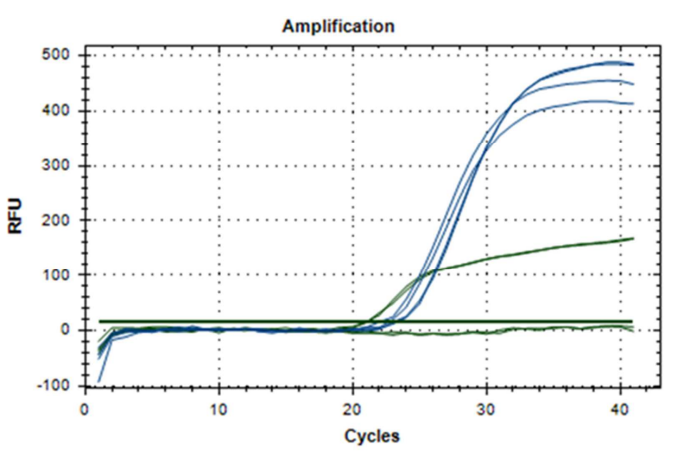

$1: 1$

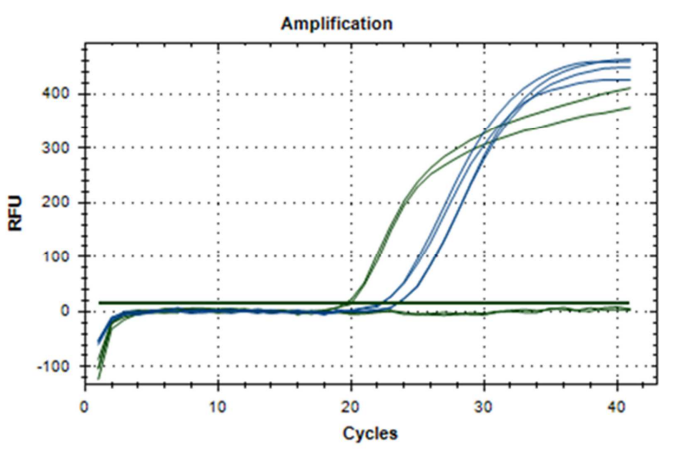

$1: 3$

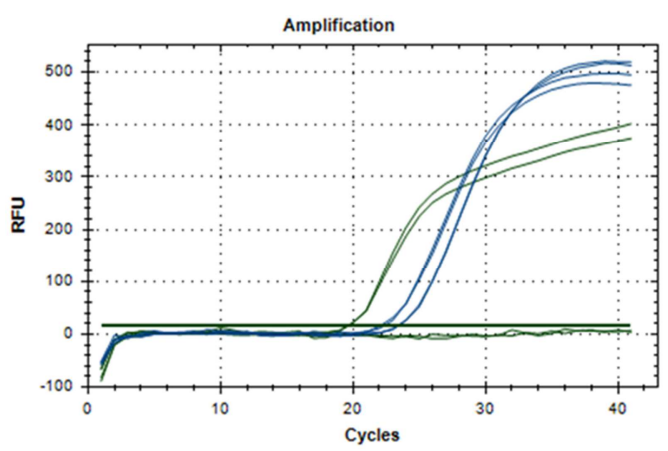

$1: 2$

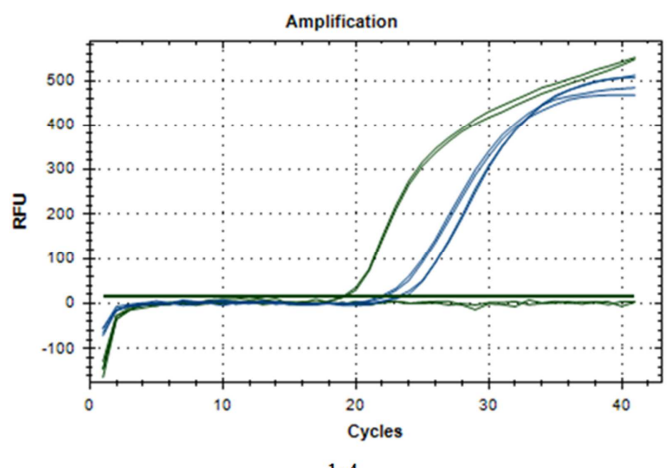

$1: 4$

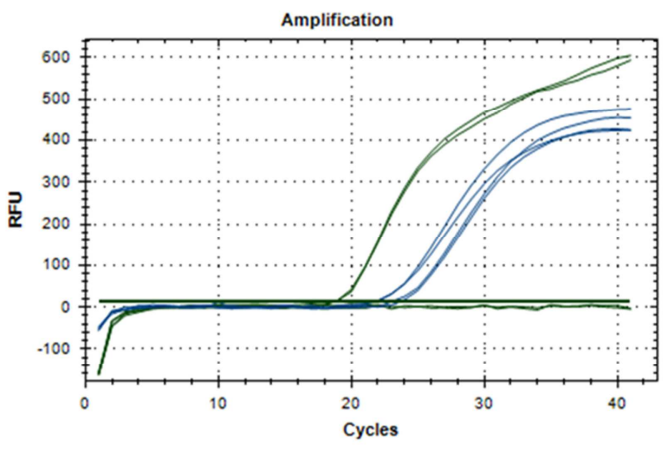

$1: 5$

(RFU: Relative Fluorescence Unit)

Figure 2. The curve diagram of duplex fluorescence PCR. 
Table 3. The Ct value and fluorescence intensity of duplex fluorescence PCR for specific cpDNA in japonica rice and endogenous gos gene.

\begin{tabular}{|c|c|c|c|c|c|}
\hline \multirow{2}{*}{$\begin{array}{l}\text { Ratio of primer and probe in } \\
\text { duplex fluorescence PCR mixture }\end{array}$} & \multirow{2}{*}{$\begin{array}{l}\text { Indicaljaponica } \\
\text { rice sample }\end{array}$} & \multicolumn{2}{|c|}{ Gos gene in rice } & \multicolumn{2}{|c|}{ Specific cpDNA in japonica rice } \\
\hline & & Ct value & Fluorescence intensity & Ct value & $\begin{array}{l}\text { Fluorescence } \\
\text { intensity }\end{array}$ \\
\hline \multirow{4}{*}{$1: 1$} & \multirow{2}{*}{ indica } & 22.67 & 400 & - & - \\
\hline & & 21.94 & 440 & - & - \\
\hline & \multirow{2}{*}{ japonica } & 22.99 & 480 & 21.04 & 165 \\
\hline & & 23.28 & 480 & 21.00 & 165 \\
\hline \multirow{4}{*}{$1: 2$} & \multirow{2}{*}{ indica } & 22.28 & 480 & - & - \\
\hline & & 22.01 & 500 & - & - \\
\hline & \multirow[b]{2}{*}{ japonica } & 23.37 & 510 & 19.60 & 380 \\
\hline & & 23.28 & 510 & 19.51 & 400 \\
\hline \multirow{4}{*}{$1: 3$} & \multirow{2}{*}{ indica } & 22.32 & 420 & - & - \\
\hline & & 22.19 & 460 & - & - \\
\hline & \multirow{2}{*}{ japonica } & 23.45 & 460 & 19.53 & 400 \\
\hline & & 23.47 & 450 & 20.04 & 370 \\
\hline \multirow{4}{*}{$1: 4$} & \multirow{2}{*}{ indica } & 21.78 & 480 & - & - \\
\hline & & 22.17 & 470 & - & - \\
\hline & \multirow{2}{*}{ japonica } & 23.49 & 500 & 19.13 & 540 \\
\hline & & 23.12 & 500 & 19.03 & 540 \\
\hline \multirow{4}{*}{$1: 5$} & \multirow{2}{*}{ indica } & 21.86 & 420 & - & - \\
\hline & & 22.02 & 470 & - & - \\
\hline & \multirow{2}{*}{ japonica } & 23.16 & 450 & 18.84 & 600 \\
\hline & & 23.77 & 420 & 18.85 & 600 \\
\hline
\end{tabular}

\subsubsection{Duplex Fluorescence PCR Procedure}

Initial denaturing at $95^{\circ} \mathrm{C}$ for $30 \mathrm{~s} ; 40$ cycles with two steps: $95^{\circ} \mathrm{C}$ for $5 \mathrm{~s}, 60^{\circ} \mathrm{C}$ for $34 \mathrm{~s}$.

\section{Results}

\subsection{Optimization and Establishment of Duplex Fluorescence PCR}

According to the results of optimization, two primers dosage proportions 1:3 and 1:4 were the best, and 1:3 was chosen to save primers within the five proportions design of primers dosage from $1: 1$ to $1: 5$ as shown in figure 2 and table 3 . The other compositions and PCR thermal cycles are as shown in table 2 and 2.2.3., to establish the method of duplex fluorescence PCR.

\subsection{Identification of Indica and Japonica Rice by Duplex Fluorescence PCR}

The results of duplex fluorescence PCR of cpDNA marker

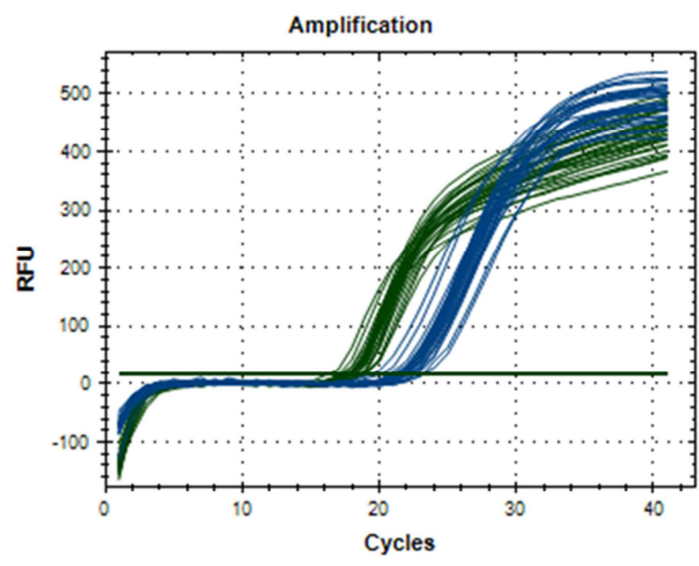

A: Two curve results (japonica type) and endogenous gos gene indicated that single curve results with positive of gos gene in indica rice and two curve results with positive of cpDNA marker and gos gene in japonica rice shown as Figure 3.

The accuracy of the duplex fluorescence PCR method was validated by 547 samples of rice seed and eaten rice. In 177 of japonica samples, 170 samples with both positive results of cpDNA marker and gos gene (Figure 3/A), a coincidence rate of $96.05 \%$ with japonica rice, and 7 samples with positive results of gos gene and negative of cpDNA marker, and non-coincidence rate of $3.95 \%$ with japonica rice, were detected. Of the 370 samples of indica rice seed and eaten rice, with positive results of gos gene and negative of cpDNA marker (Figure 3/B) were detected in 340 samples, the rate of coincidence to indica rice was $91.89 \%$. Other 30 samples with both positive results of cpDNA marker and gos gene, were detected, and the non-coincidence rate with indica was $8.11 \%$. Above results were shown as Table 4 .

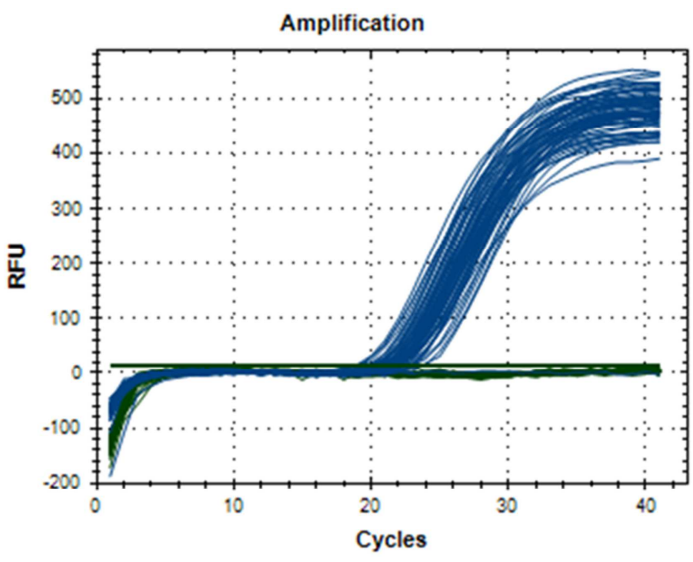

B: Single curve results (indica type)

Figure 3. The results of duplex fluorescence PCR of cpDNA marker and gos endogenous gene for identification of indica-japonica rice. 
Table 4. Identification results of indica and japonica subspecies by duplex fluorescence PCR in 547 rice samples.

\begin{tabular}{llll}
\hline Subspecie type & cpDNA deletion type (\%) & cpDNA non-deletion type (\%) & Total samples \\
\hline indica & $340(91.89 \%)$ & $30(8.11 \%)$ & 370 \\
japonica & $7(3.95 \%)$ & $170(96.05 \%)$ & 177 \\
\hline
\end{tabular}

\section{Discussions}

In this paper, based on the difference of 69 bp length between indica rice and japonica rice in the ORF100 region of PstI-12 fragment of cpDNA [23], a duplex fluorescence PCR method was established by using a previously designed primers [31]/a newly designed probe for PCR amplification of 204 bp cpDNA fragment from japonica rice and primers/probe [32] for rice endogenous gos gene amplification, to avoid the false negative results caused by DNA extraction error and to improve the accuracy of the detection results. The method was validated in the identification of 547 rice samples of known indica and japonica varieties, and the results were highly consistent with those of indica and japonica varieties, and the coincidence rate of japonica subspecies was higher than that of indica rice. The results showed that the original local varieties of japonica rice from high altitude and remote areas such as Guangxi Province, Yunnan Province, Guizhou Province had the highest coincidence rate with cpDNA, almost $100 \%$. The lowest coincidence rate was found in hybrid combinations of indica rice with complex genetic background. According to the database of China Rice Data Center, 22 of the 30 samples with non-delection cpDNA fragments expected to be indica rice had the maternal lineage of japonica rice. The results of fluorescence PCR identification of cpDNA did not accord with the expected subspecies of indica or japonica rice. The possible reasons are the traditional method of false name, natural hybridization, artificial hybridization and multigeneration backcross for nucleus replacement to improve varieties, but the cytoplasm of indica and japonica rice remains basically unchanged. In addition, it is also related to the different geographical distribution of indica and japonica subspecies. indica rice is cultivated in a wide area, with various varieties and frequent exchanges of genetic information. Especially, it is common to breed female sterile lines for hybrid rice seed production through indica-japonica crossing. japonica rice is mainly distributed in high altitude, high latitude cold and relatively closed area [14, 17], which leads to less communication of genetic information and low mutation frequency, so their coincidence rate of cpDNA detection by PCR is higher. Cai X. X. et al.(2006) had ever studied on indica-japonica differentiation of rice by DNA Insertion/Deletions of differential fragments. Their results indicated that introgression hybridization was found between indica and japonica subspecies in the area of mixed cultivation of two subspecies rice, some indica varieties has the genetic lineage of japonica rice, although the indica rice cultivars showed obvious indica phenotypic traits, they still retained some characteristic loci of japonica rice at the molecular level [15]. These results are consistent with the results of present paper. Many to hundreds of DNA loci had been used to detect in the published PCR method based on nuclear DNA $[9,18]$ and chloroplast and mitochondrial DNA [21-23], their accuracy is not ideal, usually between $80-90 \%$, and is also better in japonica than in indica subspecies. The method of indica-japonica rice identification established in this paper has a high accuracy for local conventional varieties, with more economical, more accurate and more efficient and stable, because of detecting only one cpDNA locus, but there is still much room for improvement for hybrid combinations, which needs technical improvement. Due to genetic introgression between indica and japonica rice, it is very difficult to identify with $100 \%$ accuracy using only one gene locus. So it is expected to further screen the specific key gene sites of indica-japonica subspecies for PCR amplification, and complement the method based on chloroplast DNA markers to improve the accuracy of identification results.

\section{Conclusions}

The duplex fluorescence PCR method to identify two subspecies of indica and japonica in Asia cultivated rice was developed. The accuracy of the duplex fluorescence PCR method was validated by 547 samples of rice seed and eaten rice. The coincidence rate of $96.05 \%$ was agreement with japonica rice in both fluorescence PCR positive results of cpDNA marker and gos gene. In the results with positive of gos gene and negative of cpDNA marker detected, the coincidence rate of $91.89 \%$ was agreement with indica rice. Above duplex fluorescence PCR method can be used to identify indica and japonica subspecies rice, especially suitable for the identification of conventional rice varieties.

\section{Acknowledgements}

We would like to thank the agriculture guiding (key) project (2019N0026) of Fujian Science and Technology Program for providing financial support for this project.

\section{References}

[1] Kato, S., Kosaka, H. \& Hara, S. (1928). On the affinity of rice varieties as shown by the fertility of rice plants (in Japanese). Central Agriculture Institute of Kyushu Imperial University, 2: $241-276$.

[2] Ting Y. (1949). The origin of Chinese rice culture (in Chinese). Agronomy Bulletin of College of Agriculture of Chungsan University, 7: 11-24.

[3] Ting Y. (1957). Origin and evolution of cultivated rice in China. Journal of Agriculture, 8 (3): 243-260. 
[4] Cheng, K. S. (1993). Identification of indica-japonica subspecies in Asian rice. Kunming: Yunnan Science and Technology Press, 1-23.

[5] Chen, W. B. (1999). A Review of genetic studies on the origin and differentiation of Asian cultivated rice (Oryza sativa L.), Acta Agriculturae Shanghai, 15 (3): 42-48.

[6] Ling, Q. H., Zhang, H. C. \& Ding, Y. F. (2013). Discussion of naming for two subspecies of Oryza sativa L. Scientia Agricultura Sinica, 46 (2): 250-256.

[7] Chen, W-B., Sato, Y. I., Nakamura, I. \& Nakai, H. (1994) Indica-japonica differentiation in Chinese rice landraces. Euphytica, 74 (3): 195-201.

[8] Tang, S. X., Jiang, Y. Z., Wei, X. H., Li, Z. C. \& Yu, H. Y. (2002). Genetic Diversity of isozymes of cultivated rice in China. Acta Agronomica Sinica, 28 (2): 203-207.

[9] Zhang, Q. F., Saghai, M. A. \& Lu, T. Y. (1992). Genetic diversity and differentiation of indica and japonica rice detected by RFLP analysis. Theoretical and Applied Genetics, 83: 495-499.

[10] Zhuang J. Y. Qian H. R., Lin H. X., Lu J., Cheng, S. H., Ying, C. S., Luo, L. J., Zhu, X. D., Dong, F. G., Min, S. K., Sun, Z. X., \& Z heng K. L. (1995). RFLP based Analysis of the Origin and differentiation of Oryza sativa L. Chinese Journal of Rice Sciences, 9 (3): 135-140.

[11] Sun, C. Q., Wang, X. K. \& Yoshimura, A. (1997). RFLP Analysis of nuclear DNA in common wild rice (O. rufipogon griff.) and cultivated rice (O. sativa L.). Scientia Agricultura Sinica, 30 (4): 37-44.

[12] Long, W. H. \& Xu, M. H. (2002). RAPD-based genetic difference between indica rice and japonica rice. Journal of Yunnan Agricultural University, 17 (3): 245-247.

[13] Yamanaka, S., Nakamura, I., Nakai, H., \& Sato, Y. I. (2003). Dual origin of the cultivated rice based on molecular markers of newly collected annual and perennial strains of wild rice species, Oryza nivara and O. rufipogon. Genetic Resources and Crop Evolution, 50: 529-539.

[14] Xu, J. X., Wang, Y. Y., \& Yao C. (2012). Indica-japonica differentiation and genetic variation of upland rice varieties from Yunnan Province, China. Chinese Science Bulletin, 57 (28-29): 2705-2714.

[15] Cai, X. X., Liu, J., Qiu, Y. Q., Zhao, W., Song, Z. P. \& Lu, B. R. (2006). Differentiation of indica-japonica rice revealed by insertion/deletion fragments obtained from comparative genomic study of DNA sequences between 93-11 (indica) and nipponbare (japonica). Journal of Fudan University (Natural Science), 45 (3): 309-315.

[16] Zhao W., Xia H. B., Zang S. J., Cai, X. X., \& Lu, B. R. (2008) Differentiation of Oryza species revealed by the indicajaponica. Journal of Fudan University (Natural Science), 47 (3): 281-287.

[17] Xiong, Z. Y., Zhang, S. J., Wang, Y. Y., Brian V. Ford-Lloyd, Tu, M., Jin, X., Wu, Y., Yan, H. X., Yang X., Liu, P. \& Lu, B. R. (2010). Differentiation and distribution of indica and japonica rice varieties along the altitude gradients in Yunnan Province of China as revealed by InDel molecular markers. Genetic Resources and Crop Evolution, 57: 891-902.

[18] Liu, P., Cai, X. X. \& Lu, B. R. (2012). Single-seeded InDel fingerprints in rice: An effective tool for indica-japonica rice classification and evolutionary studies. Journal of Systematic and Evolution. 50: 1-11.

[19] Chen, W. B., Nakamura, I., SATO, Y.-I. \& Nakai, H. (1993). Distribution of deletion type in cpDNA of cultivated and wild rice [J]. Japanese Journal of Genetics, 68 (6): 579-603.

[20] Yang, J. Wang, J., Can, Q., Chen, Z. D., Tang L. H., Wang Y. P., Fang X. W., Wang C. L. \& Zhong, W. G. (2009). Indicajaponica differentiation of chloroplast DNA of weedy rice in the Changjiang and Huaihe River Valley of China. Chinese Journal of Rice Sciences, 23 (4): 391-397.

[21] Wang, R. S., Wei, X., Cao, L. R., Qiao, W. H., Zhang, W. X., \& Yang, Q. W. (2011). Origin and evolution of cultivated rice (O. sativa L.) in China based on gene diversity of chloroplast genome. Journal of Plant Genetic Resources, 12 (5): 686-693.

[22] Cao, L. R., Wei, X., Huang, J., Qiao, W. H., Zang, W. X. \& Yang, Q. E. (2013). Study on the origin and evolution of Asian cultivated rice based on gene fragment nucleotides diversity of mitochondrial genome. Journal of Plant Genetic Resources, 14 (1): 18-24.

[23] Kanno, A., Watanabe, N., Nakamura, I. \& Hirai, A. (1993). Variations in chloroplast DNA from rice (Oryza sativa): differences between deletions mediated by short direct-repeat sequences within a single species. Theoretical and Applied Genetics, 86: 579-584.

[24] Rong, Q. Y., Chen, X. X., Liu, Y. N., Xu, Z., Ding, Y. F., Wu, Y. L., Ji, X. F., Zhang, Y. \& Li, B. Q. (2018). Establishment of a duplex fluorescence quantitative PCR technique for detection of Helicobacter pylori with TaqMan MGB probes. Journal of Pathogen Biology, 6: 572-574, 579.

[25] Ma, L., Yao, G. Z., Wang, Z., Meng, Q. F., Li, Y., Xiao, F., Liu, J. H., \& Wang, W. L. (2020). Establishment of duplex fluorescence RT-PCR method for detection of H5N8 subtype avian influenza virus HA and NA genes. ProgressinVeterinaryMedicine, 41 (6): 32-37.

[26] Shao M. L., Dong X., Zhao Y. L., Kong, B. H. \& Liu, S. G.. (2013). A duplex fluorescence quantitative PCR assay for detecting Listeriamonocytogenes and Staphylococcus aureus. Food Science, 34 (16): 169-172.

[27] Yang, L. L., Sun, L. X., Ruan, X. L., Qiu, D. Y., Chen, D. H., Cai, X. Q., \& Li, H. P. (2015). Development of a single-tube duplex real-time fluorescence method for the rapid quantitative detection of Fusarium oxysporum $\mathrm{f}$. sp. cubense race 1 (FOC1) and race 4 (FOC4) using TaqMan probes, Crop Protection, 68 (2): 27-35.

[28] Hou, D. J., Han, H. J., Hao, Z. H. \& Jiang, Y. B. (2016). The method of duplex fluorescence PCR for rapid detection of cattle-and sheep-derived ingredients in feedstuff. Heilongjiang Animal Science and Veterinary Medicine, 2: 247-250.

[29] Wang F.-J., Ye S.-D., Chen G.-W. \& Bao, Y. H. (2018). Establishment and verified of a duplex fluorescence quantitative PCR for screening of genetically modified soybeans and products. Science and Technology of Food Industry, 8: 236-239, 271.

[30] Yuan, J. J., Wei, S., Long, Y., Wu, X. Y., Li, X. \& Fu, W. (2020). Development and application of duplex real-time PCR assay for the detection of genetically modified soybean (Glycine max) MON87701 and MON87708. Journal of Agricultural Biotechnology, 28 (2): 342-348. 
[31] Chen, W. B., Shao, B. Y., Lin, Y. W., Miao, T. Y., Peng, J., Guo, J., Chen, B. \& Peng, H. Y. (2020). Identification method of hisien (indica) and keng (japonica) rice by SYBR-Green fluorescence-PCR detection for chloroplast DNA. China Port Science and Technology, 10: 17-25.
[32] Chen, H. Y., Chen, X. M., Chen, S. Y., Wang, Z. H., Li, L, Huang, W. S., Hu, X. Z., Zhu, S. F. \& Chen, H. J. (2010). Protocol of real-time polymerase chain reaction for detecting genetically modified components in rice and its derived products. Commodity Inspection Standards of the People's Republic of China, SN/T2584-2010. 